\title{
The first Darboux problem for wave equations with nonlinear dissipative term
}

\author{
Otar Jokhadze
}

\begin{abstract}
The first Darboux problem for wave equations with nonlinear dissipative term is considered. The uniqueness, local and global existence and blow-up of solutions of the problem mentioned are investigated. The paper's originality is the coalescence of two standard methods: a priori estimate of solutions in the class of continuous functions is given by energetic methods; basing on this result a priori estimate in the class of continuously differentiable functions using classical method of characteristics is obtained.
\end{abstract}

Mathematics Subject Classification. 35L71.

Keywords. Nonlinear wave equations, The first Darboux problem, Global and local solvability, Blow-up solutions, Dissipative term.

\section{Introduction}

In the plane of independent variables $x$ and $t$ consider nonlinear wave equation of the following form [1], [2, p. 57]

$$
L u:=u_{t t}-u_{x x}+g(x, t, u) u_{t}=f(x, t),
$$

where $g, f$ are given, and $u$ - unknown real functions.

Denote by $D_{T}:=\{(x, t): 0<x<t, 0<t<T\}$ triangular domain, bounded by characteristic segment $\gamma_{1, T}: x=t, 0 \leq t \leq T$ and by segments $\gamma_{2, T}: x=0,0 \leq t \leq T, \gamma_{3, T}: t=T, 0 \leq x \leq T$.

For Eq. (1.1) in domain $D_{T}$ consider the first Darboux problem on determination of solution $u(x, t)$ by boundary conditions [3, p. 228]

$$
\left.u\right|_{\gamma_{i, T}}=0, \quad i=1,2 .
$$

Note that on questions of existence, uniqueness and blow-up of solutions of initial, mixed, nonlocal and other problems posed for nonlinear hyperbolic type equations there are devoted a number of papers (see, e.g. [1-18]). In linear case, i. e. when $g(x, t, u)=g(x, t)$, problem (1.1), (1.2), as it is known, is wellposed and a global solvability takes place in corresponding function spaces 
(see, e.g. $[3,19]$ ). In previous literature $[1,2,4-13]$, basically are studied the Cauchy initial and mixed initial-boundary value problems in functional spaces different from spaces considered in this paper. Moreover, in papers $[1,2]$ are considered the particular cases when $g(x, t, s)=|s|^{\rho}$. In papers [14-18] there are considered the similar boundary value conditions, but for hyperbolic differential equations with nonlinear source terms.

Below we show that for certain requirements for nonlinear function $g(x, t, s)$ with respect to variable $s$ problem (1.1), (1.2) is locally solvable; the conditions for global solvability are obtained, violation of which, generally speaking, can cause a blow-up within finite interval of time.

Definition 1.1. Let $f \in C\left(\bar{D}_{T}\right), g \in C\left(\bar{D}_{T} \times \mathbb{R}\right), \mathbb{R}:=(-\infty,+\infty)$. Function $u$ is called a strong generalized solution of problem (1.1), (1.2) of class $C^{1}$ in domain $D_{T}$, if $u \in C^{1}\left(\bar{D}_{T}\right)$ and there exists such the sequence of functions $u_{n} \in$ $\stackrel{\circ}{C}^{2}\left(\bar{D}_{T}, \Gamma_{T}\right)$, that $u_{n} \rightarrow u$ and $L u_{n} \rightarrow f$ for $n \rightarrow \infty$ in spaces $C^{1}\left(\bar{D}_{T}\right)$ and $C\left(\bar{D}_{T}\right)$, respectively, where $\stackrel{\circ}{C}^{2}\left(\bar{D}_{T}, \Gamma_{T}\right):=\left\{v \in C^{2}\left(\bar{D}_{T}\right):\left.v\right|_{\Gamma_{T}}=0\right\}, \Gamma_{T}:=$ $\gamma_{1, T} \cup \gamma_{2, T}$

Remark 1.1. It is clear that if $u \in \stackrel{\circ}{C}^{2}\left(\bar{D}_{T}, \Gamma_{T}\right)$ is a classical solution of problem (1.1), (1.2), then it is a strong generalized solution of this problem of class $C^{1}$ in domain $D_{T}$. In turn, if a strong generalized solution of problem (1.1), (1.2) of class $C^{1}$ in domain $D_{T}$ belongs to space $C^{2}\left(\bar{D}_{T}\right)$, then it also is a classical solution of the problem.

Definition 1.2. Let $f \in C\left(\bar{D}_{\infty}\right), g \in C\left(\bar{D}_{\infty} \times \mathbb{R}\right)$. We say that problem (1.1), (1.2) is globally solvable in the class $C^{1}$, if for any finite $T>0$ it has a strong generalized solution of the class $C^{1}$ in domain $D_{T}$.

The paper is organized as follows. In Sect. 2 it is obtained a priori estimates of the solution of problem (1.1), (1.2) in spaces $C\left(\bar{D}_{T}\right)$ and $C^{1}\left(\bar{D}_{T}\right)$. In Sect. 3 problem (1.1), (1.2) is equivalently reduced to the system of nonlinear Volterra type integral equations and the local solvability of this problem is proved. In Sect. 4 it is shown the global solvability of the considered problem, and in Sect. 5 the uniqueness of the solution of the problem is proved. Finally, in the last Sect. 6 the nonexistence of global solvability of this problem is shown.

\section{A priori estimates of the solution of problem (1.1), (1.2) in spaces $C\left(\bar{D}_{T}\right)$ and $C^{1}\left(\bar{D}_{T}\right)$}

Lemma 2.1. Let $f \in C\left(\bar{D}_{T}\right), g \in C\left(\bar{D}_{T} \times \mathbb{R}\right)$ and

$$
g(x, t, s) \geq-M_{T},(x, t, s) \in \bar{D}_{T} \times \mathbb{R}, M_{T}:=\text { const }>0 .
$$

Then for a strong generalized solution of problem (1.1), (1.2) of class $C^{1}$ in domain $D_{T}$ it is valid the following a priori estimate

$$
\|u\|_{C\left(\bar{D}_{T}\right)} \leq c_{0}\|f\|_{C\left(\bar{D}_{T}\right)}
$$

with positive constant $c_{0}=c_{0}\left(T, M_{T}\right)$, not dependent on $u$ and $f$. 
Proof. Let $u$ be a strong generalized solution of problem (1.1), (1.2) of class $C^{1}$ in domain $D_{T}$. Then due to Definition 1.1 there exists the sequence of functions $u_{n} \in \stackrel{\circ}{C}^{2}\left(\bar{D}_{T}, \Gamma_{T}\right)$, such that

$$
\lim _{n \rightarrow \infty}\left\|u_{n}-u\right\|_{C^{1}\left(\bar{D}_{T}\right)}=0, \quad \lim _{n \rightarrow \infty}\left\|L u_{n}-f\right\|_{C\left(\bar{D}_{T}\right)}=0
$$

and therefore

$$
\lim _{n \rightarrow \infty}\left\|g\left(x, t, u_{n}\right) u_{n t}-g(x, t, u) u_{t}\right\|_{C\left(\bar{D}_{T}\right)}=0 .
$$

Consider function $u_{n} \in \stackrel{\circ}{C}^{2}\left(\bar{D}_{T}, \Gamma_{T}\right)$, as a solution of the following problem

$$
\begin{aligned}
& L u_{n}=f_{n}, \\
& \left.u_{n}\right|_{\Gamma_{T}}=0 .
\end{aligned}
$$

Here

$$
f_{n}:=L u_{n} .
$$

Multiplying the both sides of equality $(2.5)$ by $u_{n t}$ and integrating the received in domain $D_{\tau}:=\left\{(x, t) \in D_{T}: 0<t<\tau\right\}, 0<\tau \leq T$ we have $\frac{1}{2} \int_{D_{\tau}}\left(u_{n t}^{2}\right)_{t} d x d t-\int_{D_{\tau}} u_{n x x} u_{n t} d x d t+\int_{D_{\tau}} g\left(x, t, u_{n}\right) u_{n t}^{2} d x d t=\int_{D_{\tau}} f_{n} u_{n t} d x d t$.

Assume that $\Omega_{\tau}:=\bar{D}_{\infty} \cap\{t=\tau\}, 0<\tau \leq T$. Then by virtue of (2.6), integrating by parts the left-hand side of the last equality, we obtain

$$
\begin{aligned}
\int_{D_{\tau}} f_{n} u_{n t} d x d t= & \frac{1}{2} \int_{\gamma_{1, \tau}} \nu_{t}^{-1}\left[\left(u_{n x} \nu_{t}-u_{n t} \nu_{x}\right)^{2}+u_{n t}^{2}\left(\nu_{t}^{2}-\nu_{x}^{2}\right)\right] d s \\
& +\frac{1}{2} \int_{\Omega_{\tau}}\left(u_{n x}^{2}+u_{n t}^{2}\right) d x+\int_{D_{\tau}} g\left(x, t, u_{n}\right) u_{n t}^{2} d x d t
\end{aligned}
$$

where $\nu:=\left(\nu_{x}, \nu_{t}\right)$ is unit vector of outer normal to $\partial D_{\tau}$ and $\gamma_{1, \tau}:=\gamma_{1, T} \cap$ $\{t \leq \tau\}$

Taking into account the fact that operator $\nu_{t} \frac{\partial}{\partial x}-\nu_{x} \frac{\partial}{\partial t}$ is an interior differential operator on $\gamma_{1, T}$, due to (2.6) we receive

$$
\left.\left(u_{n x} \nu_{t}-u_{n t} \nu_{x}\right)\right|_{\gamma_{1, \tau}}=0 .
$$

Further, it is clear that

$$
\left.\left(\nu_{t}^{2}-\nu_{x}^{2}\right)\right|_{\gamma_{1, \tau}}=0
$$

Therefore, by virtue of (2.8)-(2.10) we get

$$
w_{n}(\tau):=\int_{\Omega_{\tau}}\left(u_{n x}^{2}+u_{n t}^{2}\right) d x \leq 2 \int_{D_{\tau}} f_{n} u_{n t} d x d t+2 M_{T} \int_{D_{\tau}} u_{n t}^{2} d x d t .
$$

Taking into account inequality

$$
2 f_{n} u_{n t} \leq u_{n t}^{2}+f_{n}^{2},
$$


due to (2.11) we have

$$
w_{n}(\tau) \leq\left(1+2 M_{T}\right) \int_{D_{\tau}} u_{n t}^{2} d x d t+\int_{D_{\tau}} f_{n}^{2} d x d t .
$$

According to the form of function $w_{n}(\tau)$ it follows that

$$
w_{n}(\tau) \leq m_{T} \int_{0}^{\tau} w_{n}(\sigma) d \sigma+\left\|f_{n}\right\|_{L_{2}\left(D_{\tau}\right)}^{2}, \quad m_{T}:=1+2 M_{T} .
$$

Whence, having the fact that the value $\left\|f_{n}\right\|_{L_{2}\left(D_{\tau}\right)}^{2}$, as a function of $\tau$ is non-decreasing, by the Gronwall's lemma [20, p. 13] we receive

$$
w_{n}(\tau) \leq \exp \left(m_{T} \tau\right)\left\|f_{n}\right\|_{L_{2}\left(D_{\tau}\right)}^{2} .
$$
is valid

If $(x, t) \in \bar{D}_{T}$, then by virtue of the condition (2.6) the following equality

$$
u_{n}(x, t)=u_{n}(x, t)-u_{n}(0, t)=\int_{0}^{x} u_{n x}(\sigma, t) d \sigma,
$$

thus due to (2.12) we have

$$
\begin{gathered}
\left|u_{n}(x, t)\right|^{2} \leq \int_{0}^{x} d \sigma \int_{0}^{x} u_{n x}^{2}(\sigma, t) d \sigma \leq x \int_{\Omega_{t}} u_{n x}^{2}(\sigma, t) d \sigma \leq x w_{n}(t) \leq t w_{n}(t) \\
\leq T \exp \left(m_{T} T\right)\left\|f_{n}\right\|_{C\left(\bar{D}_{T}\right)}^{2} m e s D_{T}=2^{-1} T^{3} \exp \left(m_{T} T\right)\left\|f_{n}\right\|_{C\left(\bar{D}_{T}\right)}^{2} .
\end{gathered}
$$

From this it follows that

$$
\left\|u_{n}\right\|_{C\left(\bar{D}_{T}\right)} \leq T \sqrt{\frac{T}{2}} \exp \left(\frac{m_{T} T}{2}\right)\left\|f_{n}\right\|_{C\left(\bar{D}_{T}\right)} .
$$

Passing in this inequality to limit for $n \rightarrow \infty$, and due to (2.3), (2.7), we have

$$
\|u\|_{C\left(\bar{D}_{T}\right)} \leq T \sqrt{\frac{T}{2}} \exp \left(\frac{m_{T} T}{2}\right)\|f\|_{C\left(\bar{D}_{T}\right)} .
$$

This proves estimate (2.2).

Remark 2.1. From (2.14) it follows that constant $c_{0}$ in estimate (2.2) can be taken

$$
c_{0}:=T \sqrt{\frac{T}{2}} \exp \left(\frac{m_{T} T}{2}\right) .
$$

Below, taking into account estimate (2.2) and using the classical method of characteristics, we receive a priori estimate in space $C^{1}\left(\bar{D}_{T}\right)$ for a strong generalized solution $u$ of problem (1.1), (1.2) of class $C^{1}$ in domain $D_{T}$.

Lemma 2.2. Under the conditions of Lemma 2.1 for a strong generalized solution u of problem (1.1), (1.2) of class $C^{1}$ in domain $D_{T}$ it is valid the following a priori estimate

$$
\|u\|_{C^{1}\left(\bar{D}_{T}\right)} \leq c_{1}
$$

with positive constant $c_{1}=c_{1}\left(T, c_{0},\|f\|_{C\left(\bar{D}_{T}\right)}\right)$, not dependent on $u$, where $\|u\|_{C^{1}\left(\bar{D}_{T}\right)}:=\max \left\{\|u\|_{C\left(\bar{D}_{T}\right)},\left\|u_{x}\right\|_{C\left(\bar{D}_{T}\right)},\left\|u_{t}\right\|_{C\left(\bar{D}_{T}\right)}\right\}$. 
Proof. Let $u$ be a strong generalized solution of the problem (1.1), (1.2) of the class $C^{1}$ in the domain $D_{T}$. Then the limit equalities $(2.3),(2.4)$ are valid, where $u_{n}$ can be considered as a solution of problem $(2.5),(2.6)$ with $f_{n}$ given by $(2.7)$.

Denote

$$
l_{i}:=\frac{\partial}{\partial t}+(-1)^{i} \frac{\partial}{\partial x}, \quad i=1,2 .
$$

For fixed natural number $n$ let us introduce the following functions

$$
v_{i n}:=l_{i} u_{n}, \quad v_{3 n}:=u_{n}, \quad i=1,2,
$$

which by taking into account (2.2) satisfy the following boundary conditions

$$
v_{1 n}(0, t)=-v_{2 n}(0, t), \quad v_{2 n}(t, t)=0, \quad v_{3 n}(t, t)=0, \quad 0 \leq t \leq T .
$$

With respect to unknown functions $v_{i n}, i=1,2,3$ by virtue of (1.1) and (2.17) we have the following system of first order partial differential equations

$$
\left\{\begin{array}{l}
l_{2} v_{1 n}=f_{n}(x, t)-\frac{1}{2} g\left(x, t, v_{3 n}\right)\left(v_{1 n}+v_{2 n}\right), \\
l_{1} v_{2 n}=f_{n}(x, t)-\frac{1}{2} g\left(x, t, v_{3 n}\right)\left(v_{1 n}+v_{2 n}\right) \\
l_{1} v_{3 n}=v_{1 n}
\end{array}\right.
$$

Integrating the received system along the corresponding characteristic curves, due to boundary conditions (2.18), we have

$$
\left\{\begin{array}{l}
v_{1 n}(x, t)-v_{1 n}(0, t)=\int_{t-x}^{t}\left(f_{n}\left(P_{\tau}\right)-\frac{1}{2} g\left(P_{\tau}, v_{3 n}\left(P_{\tau}\right)\right)\left(v_{1 n}\left(P_{\tau}\right)+v_{2 n}\left(P_{\tau}\right)\right)\right) d \tau \\
v_{2 n}(x, t)=\int_{\frac{x+t}{2}}^{t}\left(f_{n}\left(Q_{\tau}\right)-\frac{1}{2} g\left(Q_{\tau}, v_{3 n}\left(Q_{\tau}\right)\right)\left(v_{1 n}\left(Q_{\tau}\right)+v_{2 n}\left(Q_{\tau}\right)\right)\right) d \tau, \\
v_{3 n}(x, t)=\int_{\frac{x+t}{2}}^{t^{t}} v_{1 n}\left(Q_{\tau}\right) d \tau
\end{array}\right.
$$

where $P_{\tau}:=(x-t+\tau, \tau), Q_{\tau}:=(x+t-\tau, \tau)$.

From the second equation of the received system and the first equality of (2.18), taking into account notation $Q_{\tau_{0}}:=(t-\tau, \tau)$ this system can be rewritten as follows

$$
\left\{\begin{array}{l}
v_{1 n}(x, t)=-\frac{1}{2} \int_{t-x}^{t} g\left(P_{\tau}, v_{3 n}\left(P_{\tau}\right)\right)\left(v_{1 n}\left(P_{\tau}\right)+v_{2 n}\left(P_{\tau}\right)\right) d \tau \\
\quad+\frac{1}{2} \int_{\frac{t}{2}}^{t} g\left(Q_{\tau_{0}}, v_{3 n}\left(Q_{\tau_{0}}\right)\right)\left(v_{1 n}\left(Q_{\tau_{0}}\right)+v_{2 n}\left(Q_{\tau_{0}}\right)\right) d \tau+F_{1 n}(x, t) \\
v_{2 n}(x, t)=-\frac{1}{2} \int_{\frac{x+t}{2}}^{t} g\left(Q_{\tau}, v_{3 n}\left(Q_{\tau}\right)\right)\left(v_{1 n}\left(Q_{\tau}\right)+v_{2 n}\left(Q_{\tau}\right)\right) d \tau+F_{2 n}(x, t), \\
v_{3 n}(x, t)=\int_{\frac{x+t}{2}}^{t} v_{1 n}\left(Q_{\tau}\right) d \tau
\end{array}\right.
$$

Here

$$
F_{1 n}(x, t):=\int_{t-x}^{t} f_{n}\left(P_{\tau}\right) d \tau-\int_{\frac{t}{2}}^{t} f_{n}\left(Q_{\tau_{0}}\right) d \tau, \quad F_{2 n}(x, t):=\int_{\frac{x+t}{2}}^{t} f_{n}\left(Q_{\tau}\right) d \tau .
$$


By passing in equalities (2.20), (2.21) to limit as $n \rightarrow \infty$ in the space $C\left(\bar{D}_{T}\right)$ and taking into account $(2.3),(2.4),(2.7)$ and $(2.17)$ we have

$$
\left\{\begin{array}{l}
v_{1}(x, t)=-\frac{1}{2} \int_{t-x}^{t} g\left(P_{\tau}, v_{3}\left(P_{\tau}\right)\right)\left(v_{1}\left(P_{\tau}\right)+v_{2}\left(P_{\tau}\right)\right) d \tau \\
\quad+\frac{1}{2} \int_{\frac{t}{2}}^{t} g\left(Q_{\tau_{0}}, v_{3}\left(Q_{\tau_{0}}\right)\right)\left(v_{1}\left(Q_{\tau_{0}}\right)+v_{2}\left(Q_{\tau_{0}}\right)\right) d \tau+F_{1}(x, t), \\
v_{2}(x, t)=-\frac{1}{2} \int_{\frac{x+t}{2}}^{t} g\left(Q_{\tau}, v_{3}\left(Q_{\tau}\right)\right)\left(v_{1}\left(Q_{\tau}\right)+v_{2}\left(Q_{\tau}\right)\right) d \tau+F_{2}(x, t), \\
v_{3}(x, t)=\int_{\frac{x+t}{2}}^{t} v_{1}\left(Q_{\tau}\right) d \tau
\end{array}\right.
$$

where $v_{i}:=\lim _{n \rightarrow \infty} v_{i n}$ (by the norm of space $C\left(\bar{D}_{T}\right)$ ), $i=1,2,3$, and

$$
F_{1}(x, t):=\int_{t-x}^{t} f\left(P_{\tau}\right) d \tau-\int_{\frac{t}{2}}^{t} f\left(Q_{\tau_{0}}\right) d \tau, \quad F_{2}(x, t):=\int_{\frac{x+t}{2}}^{t} f\left(Q_{\tau}\right) d \tau .
$$

Remark 2.2. It is clear that $v_{3}=u$, which is a strong generalized solution of the problem (1.1), (1.2) of class $C^{1}$ in domain $D_{T}$, besides

$$
v_{1}=u_{t}-u_{x}, v_{2}=u_{t}+u_{x} .
$$

Let $G_{T}:=\left\{(x, t, s) \in \mathbb{R}^{3}:(x, t) \in \bar{D}_{T},|s| \leq c_{0}\|f\|_{C\left(\bar{D}_{T}\right)}\right\}$ and

$$
K=K\left(T, c_{0},\|f\|_{C\left(\bar{D}_{T}\right)}\right):=\frac{1}{2} \sup _{(x, t, s) \in G_{T}}|g(x, t, s)|<+\infty,
$$

where $c_{0}$ is defined by $(2.15)$. Then due to a priori estimate (2.2) for a strong generalized solution $u=v_{3}$ of problem $(1.1),(1.2)$ of class $C^{1}$ in domain $D_{T}$ we receive

$$
\left|g\left(x, t, v_{3}(x, t)\right)\right| \leq 2 K, \quad(x, t) \in \bar{D}_{T} .
$$

Let

$$
\omega_{i}(t):=\sup _{(\xi, \tau) \in \bar{D}_{t}}\left|v_{i}(\xi, \tau)\right|, \quad i=1,2,3, \quad F(t):=\sup _{(\xi, \tau) \in \bar{D}_{t}}|f(\xi, \tau)| .
$$

From (2.22), due (2.23), (2.26) and (2.27) it follows that

$$
\begin{aligned}
& \left|v_{1}(x, t)\right| \leq 2 K \int_{0}^{t}\left(\omega_{1}(\tau)+\omega_{2}(\tau)\right) d \tau+2 t F(t) \\
& \left|v_{2}(x, t)\right| \leq K \int_{0}^{t}\left(\omega_{1}(\tau)+\omega_{2}(\tau)\right) d \tau+t F(t), \quad\left|v_{3}(x, t)\right| \leq \int_{0}^{t} \omega_{1}(\tau) d \tau .
\end{aligned}
$$

Whence for $(\xi, \tau) \in \bar{D}_{t}$ we receive

$$
\begin{aligned}
& \left|v_{1}(\xi, \tau)\right| \leq 2 K \int_{0}^{\tau}\left(\omega_{1}\left(\tau_{1}\right)+\omega_{2}\left(\tau_{1}\right)\right) d \tau_{1}+2 \tau F(\tau) \\
& \left|v_{2}(\xi, \tau)\right| \leq K \int_{0}^{\tau}\left(\omega_{1}\left(\tau_{1}\right)+\omega_{2}\left(\tau_{1}\right)\right) d \tau_{1}+\tau F(\tau), \quad\left|v_{3}(\xi, \tau)\right| \leq \int_{0}^{\tau} \omega_{1}\left(\tau_{1}\right) d \tau_{1} .
\end{aligned}
$$


Thus due to (2.27) and the fact that $t F(t)$ is non-decreasing function it follows that

$$
\begin{aligned}
& \omega_{1}(t) \leq 2 K \int_{0}^{t}\left(\omega_{1}(\tau)+\omega_{2}(\tau)\right) d \tau+2 t F(t) \\
& \omega_{2}(t) \leq K \int_{0}^{t}\left(\omega_{1}(\tau)+\omega_{2}(\tau)\right) d \tau+t F(t), \quad \omega_{3}(t) \leq \int_{0}^{t} \omega_{1}(\tau) d \tau .
\end{aligned}
$$

Setting that $\omega(t):=\max _{1 \leq i \leq 3} \omega_{i}(t), 0 \leq t \leq T$, from the inequalities given above it follows that

$$
\omega(t) \leq(4 K+1) \int_{0}^{t} \omega(\tau) d \tau+2 t F(t), 0 \leq t \leq T,
$$

whence by virtue of Gronwall's Lemma we have

$\omega(t) \leq 2 t F(t) \exp ((4 K+1) t) \leq 2 T \exp ((4 K+1) T)\|f\|_{C\left(\bar{D}_{T}\right)}, \quad 0 \leq t \leq T$.

Now from (2.24) it is easy to receive

$$
\|u\|_{C^{1}\left(\bar{D}_{T}\right)} \leq\|\omega\|_{C[0, T]} \leq 2 T \exp ((4 K+1) T)\|f\|_{C\left(\bar{D}_{T}\right)} .
$$

The Lemma 2.2 is proved, besides

$$
c_{1}:=2 T \exp ((4 K+1) T)\|f\|_{C\left(\bar{D}_{T}\right)},
$$

where $K$ is defined by (2.25).

\section{The equivalency of the problem (1.1), (1.2) and the system of nonlinear Volterra type integral equations (2.22) and the local solvability of the problem (1.1), (1.2)}

In Sect. 2 we have reduced the problem (1.1), (1.2) to the Volterra type system of nonlinear integral equations (2.22). Before consideration the solvability of the problem (1.1), (1.2), let us prove the following lemma.

Lemma 3.1. If a function $u \in C^{1}\left(\bar{D}_{T}\right)$ is a strong generalized solution of the problem (1.1), (1.2) of the class $C^{1}$ in the domain $D_{T}$, then $v_{1}:=l_{1} u, v_{2}:=$ $l_{2} u, v_{3}:=u$ is the continuous solution of the Volterra type system of nonlinear integral equations (2.22) and vice versa, if $v_{1}, v_{2}, v_{3}$ is the continuous solution of the system (2.22), then $u:=v_{3}$ is a strong generalized solution of the problem (1.1), (1.2) of the class $C^{1}$ in the domain $D_{T}$, and the equalities $v_{1}=l_{1} u, v_{2}=l_{2} u$ are valid.

Proof. Indeed, let $v_{1}, v_{2}, v_{3} \in C\left(\bar{D}_{T}\right)$ is the solution of the system of equations (2.22). It is clear that functions $v_{1}, v_{2}, v_{3}$ satisfy the following system of differential equations in partial derivatives of the first order

$$
l_{2} v_{1}=H, \quad l_{1} v_{2}=H, \quad l_{1} v_{3}=v_{1},
$$

where

$$
2 H:=2 f(x, t)-g\left(x, t, v_{3}\right)\left(v_{1}+v_{2}\right)
$$


Moreover, let us show that

$$
l_{2} v_{3}=v_{2} \text {. }
$$

Indeed, due to the equalities (3.1) for any test function $w \in C_{0}^{\infty}\left(D_{T}\right)$ it is valid the following equalities in the functional sense due to the distribution theory of L. Schwartz [21]:

$$
\begin{aligned}
& \left\langle l_{1}\left(v_{2}-l_{2} v_{3}\right), w\right\rangle=-\left\langle v_{2}-l_{2} v_{3}, l_{1} w\right\rangle=-\left\langle v_{2}, l_{1} w\right\rangle+\left\langle l_{2} v_{3}, l_{1} w\right\rangle \\
& \quad=\left\langle l_{1} v_{2}, w\right\rangle-\left\langle v_{3}, l_{2} l_{1} w\right\rangle=\left\langle l_{1} v_{2}, w\right\rangle-\left\langle v_{3}, l_{1} l_{2} w\right\rangle \\
& \quad=\left\langle l_{1} v_{2}, w\right\rangle+\left\langle l_{1} v_{3}, l_{2} w\right\rangle=\left\langle l_{1} v_{2}, w\right\rangle+\left\langle v_{1}, l_{2} w\right\rangle \\
& \quad=\left\langle l_{1} v_{2}, w\right\rangle-\left\langle l_{2} v_{1}, w\right\rangle=\left\langle l_{1} v_{2}-l_{2} v_{1}, w\right\rangle=0 .
\end{aligned}
$$

Whence, due to the Theorem 1.4.2 from [21] we conclude that (3.3) takes place in the classical sense. Therefore, in view of (3.1) we have $v_{3} \in C^{1}$ and

$$
v_{1}+v_{2}=2 v_{3 t} \text {. }
$$

Let us extend functions $f \in C\left(\bar{D}_{T}\right)$ and $g \in C\left(\bar{D}_{T} \times \mathbb{R}\right)$ continuously in $\mathbb{R}^{2}$ and $\mathbb{R}^{3}$ respectively, saving the same notations. Analogously extend functions $v_{i} \in C\left(\bar{D}_{T}\right), i=1,2$ and $v_{3} \in C^{1}\left(\bar{D}_{T}\right)$ with the same class of smoothness $\mathbb{R}^{2}$, not changing their notations. Then function $H$ will be extended on whole plane $\mathbb{R}^{2}[22,23]$.

Let

$$
\theta_{\varepsilon}(y)= \begin{cases}C_{\varepsilon} \exp \left[-\frac{\varepsilon^{2}}{\varepsilon^{2}-|y|^{2}}\right], & |y|<\varepsilon \\ 0, & |y| \geq \varepsilon\end{cases}
$$

where $y:=(x, t) \in \mathbb{R}^{2}$, and $C_{\varepsilon}$ positive constant, which is defined from the equality

$$
C_{\varepsilon} \varepsilon^{2} \int_{|\xi|<1} \exp \left[-\frac{1}{1-|\xi|^{2}}\right] d \xi=1, \quad \xi:=\left(\xi_{1}, \xi_{2}\right)
$$

$\varepsilon$ is any positive number, and $|\cdot|$ is the norm in the Euclidian space $\mathbb{R}^{2}$.

Consider the sequence of functions

$$
\stackrel{n}{v_{i}}:=v_{i} * \theta_{\frac{1}{n}} \in C^{\infty}\left(\mathbb{R}^{2}\right), \quad i=1,2,3, \quad \stackrel{n}{H}:=H * \theta_{\frac{1}{n}},
$$

where $*$ is the known convolution operation [21], and function $\theta_{\varepsilon}$ is defined from (3.5).

In view of the properties of convolution we have [21]

$$
\begin{gathered}
\lim _{n \rightarrow \infty}\left\|\stackrel{n}{v_{i}}-v_{i}\right\|_{C\left(\bar{D}_{T}\right)}=0, i=1,2, \lim _{n \rightarrow \infty}\left\|\stackrel{n}{v_{3}}-v_{3}\right\|_{C^{1}\left(\bar{D}_{T}\right)}=0, \\
\lim _{n \rightarrow \infty}\|\stackrel{n}{H}-H\|_{C\left(\bar{D}_{T}\right)}=0 .
\end{gathered}
$$

Below we prove that the sequence of function $u_{n}:=\left.\stackrel{n}{v}_{3}\right|_{\bar{D}_{T}} \in C^{2}\left(\bar{D}_{T}\right)$ satisfies the conditions (2.3), (2.4), (2.6) and, therefore, $u:=v_{3}$ is a strong generalized solution of the problem (1.1), (1.2) of the class $C^{1}$ in the domain $D_{T}$ due to Definition 1.1. 
According to the properties of convolution and the equalities (3.1), (3.3), functions $\stackrel{n}{v}_{i}, i=1,2,3$, defined from (3.6), satisfy the following equalities

$$
l_{2} \stackrel{n}{v_{1}}=\stackrel{n}{H}, \quad l_{1} \stackrel{n}{v_{2}}=\stackrel{n}{H}, \quad l_{1} \stackrel{n}{v_{3}}=\stackrel{n}{v_{1}}, \quad l_{2} \stackrel{n}{v_{3}}=\stackrel{n}{v_{2}} .
$$

Further, according to the definition of operator $L$ from (1.1) and the equalities (3.8) we have

$$
L \stackrel{n}{v}_{3}=l_{2} l_{1} \stackrel{n}{v}_{3}+g\left(x, t, \stackrel{n}{v}_{3}\right) \stackrel{n}{v}_{3 t}=l_{2} \stackrel{n}{v}_{1}+g\left(x, t, \stackrel{n}{v}_{3}\right) \stackrel{n}{v_{3 t}}=\stackrel{n}{H}+g\left(x, t, \stackrel{n}{v}_{3}\right) \stackrel{n}{v_{3}} .
$$

Whence due to the equalities (3.7) it follows that $L \stackrel{n}{v_{3}} \rightarrow H+g\left(x, t, v_{3}\right) v_{3 t}$ for $n \rightarrow \infty$ in the space $C\left(\bar{D}_{T}\right)$. At the same time due to (3.2), (3.4) we have $H+g\left(x, t, v_{3}\right) v_{3 t}=f$. Therefore

$$
\lim _{n \rightarrow \infty}\left\|L \stackrel{n}{v}_{3}-f\right\|_{C\left(\bar{D}_{T}\right)}=0 .
$$

Now let us verify the validity of the boundary conditions (1.2). According to the properties of convolution and the equalities $(2.21),(2.22)$ we have

$$
\stackrel{n}{v}_{3}(t, t) \rightarrow v_{3}(t, t)=0
$$

for $n \rightarrow \infty$ in the space $C\left(\gamma_{1, T}\right)$.

Analogously, due to the properties of convolution and the equalities (3.4), (2.22) and (2.23) we have

$$
\stackrel{n}{v}_{3 t}(0, \cdot) \rightarrow v_{3 t}(0, \cdot)=\frac{1}{2}\left[v_{1}(0, \cdot)+v_{2}(0, \cdot)\right]=0
$$

for $n \rightarrow \infty$ in the space $C\left(\gamma_{2, T}\right)$. Integrating the received equality with respect to the variable $t$, due to the (3.10) we obtain

$$
v_{3}(0, \cdot)=0
$$

The equalities $v_{1}=l_{1} u, v_{2}=l_{2} u$ immediately follow from above-brought considerations, which together with (3.9)-(3.11) prove Lemma 3.1, since the inverse proposition is obvious.

Now let us prove the local solvability of the system of Volterra type nonlinear integral equations (2.22).

Let

$$
f \in C\left(\bar{D}_{\infty}\right), \quad f_{\infty}:=\sup _{(x, t) \in \bar{D}_{\infty}}|f(x, t)|<+\infty, \quad g \in C\left(\bar{D}_{\infty} \times \mathbb{R}\right)
$$

and for $(x, t) \in \bar{D}_{\infty}, \quad|s|,\left|s_{1}\right|,\left|s_{2}\right| \leq R$

$$
|g(x, t, s)| \leq M(R), \quad\left|g\left(x, t, s_{2}\right)-g\left(x, t, s_{1}\right)\right| \leq c(R)\left|s_{2}-s_{1}\right|,
$$

where $M(R)$ and $c(R)$ are some non-negative continuous functions of argument $R \geq 0$.

Theorem 3.1. Let the functions $f$ and $g$ satisfy conditions (3.13), (3.14). Then there exists positive number $T_{*}:=T_{*}(f, g)$, such that for $T \leq T_{*}$ the problem (1.1), (1.2) will have at least one strong generalized solution $u$ of the class $C^{1}$ in domain $D_{T}$ 
Proof. According to Lemma 3.1 the problem (1.1), (1.2) in the space $C^{1}\left(\bar{D}_{T}\right)$ is equivalent to the system of Volterra type nonlinear integral equations (2.22) in the space $C\left(\bar{D}_{T}\right)$. Below we will prove the unique solvability of system $(2.22)$ by the principle of contracted mappings [see e. g., 24, p. 390].

Let $V:=\left(v_{1}, v_{2}, v_{3}\right)$. Consider vectorial operator $\Phi:=\left(\Phi_{1}, \Phi_{2}, \Phi_{3}\right)$, acting by the formula

$$
\left\{\begin{array}{l}
\left(\Phi_{1} V\right)(x, t)=-\frac{1}{2} \int_{t-x}^{t} g\left(P_{\tau}, v_{3}\left(P_{\tau}\right)\right)\left(v_{1}\left(P_{\tau}\right)+v_{2}\left(P_{\tau}\right)\right) d \tau \\
\quad+\frac{1}{2} \int_{\frac{t}{2}}^{t} g\left(Q_{\tau_{0}}, v_{3}\left(Q_{\tau_{0}}\right)\right)\left(v_{1}\left(Q_{\tau_{0}}\right)+v_{2}\left(Q_{\tau_{0}}\right)\right) d \tau+F_{1}(x, t) \\
\left(\Phi_{2} V\right)(x, t)=-\frac{1}{2} \int_{\frac{x+t}{2}}^{t} g\left(Q_{\tau}, v_{3}\left(Q_{\tau}\right)\right)\left(v_{1}\left(Q_{\tau}\right)+v_{2}\left(Q_{\tau}\right)\right) d \tau \\
\quad+F_{2}(x, t), \\
\left(\Phi_{3} V\right)(x, t)=\int_{\frac{x+t}{2}}^{t} v_{1}\left(Q_{\tau}\right) d \tau
\end{array}\right.
$$

Then the system (2.22) can be rewritten as follows

$$
V=\Phi V
$$

Let $\|V\|_{X_{T}}:=\max _{1 \leq i \leq 3}\left\{\left\|v_{i}\right\|_{C\left(\bar{D}_{T}\right)}\right\}, \quad V \in X_{T}:=C\left(\bar{D}_{T} ; \mathbb{R}^{3}\right)$, where $C\left(\bar{D}_{T} ; \mathbb{R}^{3}\right)$ is the set of all continuous vector-functions $V: \bar{D}_{T} \rightarrow \mathbb{R}^{3}$. Let us denote by $B_{R}:=\left\{V \in X_{T}:\|V\|_{X_{T}} \leq R\right\}$ closed ball of radius $R>0$ in Banach space $X_{T}$ with a center in the null element.

Below we prove that: (i) $\Phi$ maps a ball $B_{R}$ into itself; (ii) $\Phi$ is a contracted mapping on $B_{R}$.

Indeed, due to the first inequality of (3.14) from (3.15) for $V:\|V\|_{X_{T}} \leq R$ we have

$$
\begin{aligned}
& \left|\left(\Phi_{1} V\right)(x, t)\right| \leq 2 T\left(R M(R)+\|f\|_{C\left(\bar{D}_{T}\right)}\right), \\
& \left|\left(\Phi_{2} V\right)(x, t)\right| \leq T\left(R M(R)+\|f\|_{C\left(\bar{D}_{T}\right)}\right), \quad\left|\left(\Phi_{3} V\right)(x, t)\right| \leq T R .
\end{aligned}
$$

From these estimates it follows that

$$
\|\Phi V\|_{X_{T}} \leq 2 T\left(R M(R)+R+\|f\|_{C\left(\bar{D}_{T}\right)}\right) \leq 2 T\left(R M(R)+R+f_{\infty}\right)
$$

where $f_{\infty}$ is defined in (3.13).

Assume that the value of $T$ with fixed $R>0$ is too small that

$$
2 T\left(R M(R)+R+f_{\infty}\right) \leq R
$$

so $\Phi V \in B_{R}$ and therefore condition (i) is fulfilled. 
Further, due to (3.14) from (3.15) for $V^{i}:\left\|V^{i}\right\|_{X_{T}} \leq R, i=1,2$, we have

$$
\begin{aligned}
& \left|\left(\Phi_{1} V^{2}-\Phi_{1} V^{1}\right)(x, t)\right| \\
& \leq \frac{1}{2} \int_{t-x}^{t}\left(\left|g\left(P_{\tau}, v_{3}^{2}\left(P_{\tau}\right)\right)-g\left(P_{\tau}, v_{3}^{1}\left(P_{\tau}\right)\right)\right| \mid v_{1}^{2}\left(P_{\tau}\right)\right. \\
& \left.\quad+v_{2}^{2}\left(P_{\tau}\right)|+| g\left(P_{\tau}, v_{3}^{1}\left(P_{\tau}\right)\right)|| v_{1}^{2}\left(P_{\tau}\right)-v_{1}^{1}\left(P_{\tau}\right)+v_{2}^{2}\left(P_{\tau}\right)-v_{2}^{1}\left(P_{\tau}\right) \mid\right) d \tau \\
& \quad+\frac{1}{2} \int_{\frac{t}{2}}^{t}\left(\left|g\left(Q_{\tau_{0}}, v_{3}^{2}\left(Q_{\tau_{0}}\right)\right)-g\left(Q_{\tau_{0}}, v_{3}^{1}\left(Q_{\tau_{0}}\right)\right)\right|\left|v_{1}^{2}\left(Q_{\tau_{0}}\right)+v_{2}^{2}\left(Q_{\tau_{0}}\right)\right|\right. \\
& \left.\quad+\left|g\left(Q_{\tau_{0}}, v_{3}^{1}\left(Q_{\tau_{0}}\right)\right)\right|\left|v_{1}^{2}\left(Q_{\tau_{0}}\right)-v_{1}^{1}\left(Q_{\tau_{0}}\right)+v_{2}^{2}\left(Q_{\tau_{0}}\right)-v_{2}^{1}\left(Q_{\tau_{0}}\right)\right|\right) d \tau \\
& \leq 2 T(R c(R)+M(R))\left\|V^{2}-V^{1}\right\|_{X_{T}} .
\end{aligned}
$$

Analogously

$$
\begin{aligned}
& \left|\left(\Phi_{2} V^{2}-\Phi_{2} V^{1}\right)(x, t)\right| \leq \frac{1}{2} \int_{\frac{x+t}{2}}^{t}\left(\mid g\left(Q_{\tau}, v_{3}^{2}\left(Q_{\tau}\right)\right)-g\left(Q_{\tau}, v_{3}^{1}\left(Q_{\tau}\right)\right) \| v_{1}^{2}\left(Q_{\tau}\right)\right. \\
& \left.\quad+v_{2}^{2}\left(Q_{\tau}\right)|+| g\left(Q_{\tau}, v_{3}^{1}\left(Q_{\tau}\right)\right)|| v_{1}^{2}\left(Q_{\tau}\right)-v_{1}^{1}\left(Q_{\tau}\right)+v_{2}^{2}\left(Q_{\tau}\right)-v_{2}^{1}\left(Q_{\tau}\right) \mid\right) d \tau \\
& \leq T(R c(R)+M(R))\left\|V^{2}-V^{1}\right\|_{X_{T}}
\end{aligned}
$$

and

$$
\left|\left(\Phi_{3} V^{2}-\Phi_{3} V^{1}\right)(x, t)\right| \leq \int_{\frac{x+t}{2}}^{t}\left|v_{1}^{2}\left(Q_{\tau}\right)-v_{1}^{1}\left(Q_{\tau}\right)\right| d \tau \leq T\left\|V^{2}-V^{1}\right\|_{X_{T}} .
$$

Assume that for fixed $R>0$ number $T$ is too small that

$$
\max (T, 2 T(R c(R)+R+M(R))) \leq \frac{1}{2}<1,
$$

and thus $\left\|\Phi V^{2}-\Phi V^{1}\right\|_{X_{T}} \leq \frac{1}{2}\left\|V^{2}-V^{1}\right\|_{X_{T}}$. Thus operator $\Phi$ is a contracted mapping on set $B_{R}$, i.e. condition (ii) is fulfilled.

From (3.17) and (3.18), in turn, follows that, if $T \leq T_{*}$, where

$$
T_{*}:=\min \left\{\frac{R}{2\left(R M(R)+R+f_{\infty}\right)}, \frac{1}{2}, \frac{1}{4(R c(R)+R+M(R))}\right\},
$$

then $\|\Phi V\|_{X_{T}} \leq R$ and $\left\|\Phi V^{2}-\Phi V^{1}\right\|_{X_{T}} \leq \frac{1}{2}\left\|V^{2}-V^{1}\right\|_{X_{T}}$ for $V, V^{1}, V^{2} \in B_{R}$.

The application of the contracted mapping principle shows that there exists a solution $V$ of $(3.16)$ in $C\left(\bar{D}_{T} ; \mathbb{R}^{3}\right)$ for $0<T \leq T_{*}$. Theorem 3.1 is proved completely.

\section{The case of global solvability of the problem (1.1), (1.2)}

Theorem 4.1. Let the conditions (2.1), (3.13) and (3.14) be valid. Then for any $T>0$ the problem (1.1), (1.2) has a strong generalized solution of the class $C^{1}$ in the domain $D_{T}$. 
Proof. As it was noted in Lemma 3.1 the problem (1.1), (1.2) in the space $C^{1}\left(\bar{D}_{T}\right)$ is equivalent to the system of nonlinear integral equations (2.22) in the space $C\left(\bar{D}_{T}\right)$. In view of (3.13), (3.14) the truth of the Theorem 4.1 for sufficiently small $T$, namely for $T \leq T_{*}$, where $T_{*}$ is given by the equality (3.19) follows from the Theorem 3.1. Let now $T>T_{*}$, and $V^{T_{*}}:=\left(v_{1}^{T_{*}}, v_{2}^{T_{*}}, v_{3}^{T_{*}}\right)$ is a solution of the system of nonlinear integral equations (2.22), or, the same, of vector equation (3.16) in the domain $D_{T_{*}}$ of the space $C\left(\bar{D}_{T_{*}}\right)$ according to Theorem 3.1. For $t>\Delta t_{1}:=T_{*}$ rewrite system (2.22) as follows

$$
\left\{\begin{array}{l}
v_{1}(x, t)=-\frac{1}{2} \int_{\alpha_{1}\left(x, t, \Delta t_{1}\right)}^{t} g\left(P_{\tau}, v_{3}\left(P_{\tau}\right)\right)\left(v_{1}\left(P_{\tau}\right)+v_{2}\left(P_{\tau}\right)\right) d \tau \\
\quad+\frac{1}{2} \int_{\alpha_{2}\left(x, t, \Delta t_{1}\right)}^{t} g\left(Q_{\tau_{0}}, v_{3}\left(Q_{\tau_{0}}\right)\right)\left(v_{1}\left(Q_{\tau_{0}}\right)+v_{2}\left(Q_{\tau_{0}}\right)\right) d \tau+F_{1, \Delta t_{1}}(x, t) \\
v_{2}(x, t)=-\frac{1}{2} \int_{\alpha_{3}\left(x, t, \Delta t_{1}\right)}^{t} g\left(Q_{\tau}, v_{3}\left(Q_{\tau}\right)\right)\left(v_{1}\left(Q_{\tau}\right)+v_{2}\left(Q_{\tau}\right)\right) d \tau \\
\quad+F_{2, \Delta t_{1}}(x, t), \\
v_{3}(x, t)=\int_{\alpha_{3}\left(x, t, \Delta t_{1}\right)}^{t} v_{1}\left(Q_{\tau}\right) d \tau+F_{3, \Delta t_{1}}(x, t)
\end{array}\right.
$$

where

$$
\begin{aligned}
& \alpha_{1}\left(x, t, \Delta t_{1}\right):=\max \left(\Delta t_{1}, t-x\right), \quad \alpha_{2}\left(x, t, \Delta t_{1}\right):=\max \left(\Delta t_{1}, \frac{t}{2}\right), \\
& \alpha_{3}\left(x, t, \Delta t_{1}\right):=\max \left(\Delta t_{1}, \frac{x+t}{2}\right) \\
& \left\{\begin{array}{l}
F_{1, \Delta t_{1}}(x, t):=-\frac{1}{2} \int_{t-x}^{\alpha_{1}\left(x, t, \Delta t_{1}\right)} g\left(P_{\tau}, v_{3}^{T_{*}}\left(P_{\tau}\right)\right)\left(v_{1}^{T_{*}}\left(P_{\tau}\right)+v_{2}^{T_{*}}\left(P_{\tau}\right)\right) d \tau \\
\quad+\frac{1}{2} \int_{\frac{t}{2}}^{\alpha_{2}\left(x, t, \Delta t_{1}\right)} g\left(Q_{\tau_{0}}, v_{3}^{T_{*}}\left(Q_{\tau_{0}}\right)\right)\left(v_{1}^{T_{*}}\left(Q_{\tau_{0}}\right)+v_{2}^{T_{*}}\left(Q_{\tau_{0}}\right)\right) d \tau+F_{1}(x, t), \\
F_{2, \Delta t_{1}}(x, t):=-\frac{1}{2} \int_{\frac{x+t}{2}}^{\alpha_{3}\left(x, t, \Delta t_{1}\right)} g\left(Q_{\tau}, v_{3}^{T_{*}}\left(Q_{\tau}\right)\right)\left(v_{1}^{T_{*}}\left(Q_{\tau}\right)+v_{2}^{T_{*}}\left(Q_{\tau}\right)\right) d \tau \\
+F_{2}(x, t), \\
F_{3, \Delta t_{1}}(x, t):=\int_{\frac{x+t}{2}}^{\alpha_{3}\left(x, t, \Delta t_{1}\right)} v_{1}^{T_{*}}\left(Q_{\tau}\right) d \tau .
\end{array}\right.
\end{aligned}
$$

Since the conditions of Lemma 2.2 are fulfilled, then for any positive $\tau \leq T$ for solution of vector equation (3.16) in the domain $D_{\tau}$ of the space $C\left(\bar{D}_{\tau}\right)$ it is valid a priori estimate

$$
\|V\|_{C\left(\bar{D}_{\tau}\right)} \leq R^{T}\left(\|f\|_{C\left(\bar{D}_{\tau}\right)}\right)
$$

where $R^{T}=R^{T}(s)$ is a non-decreasing continuous function of its argument $s \geq 0$. 
Let $R_{*}:=R^{T}\left(\|f\|_{C\left(\bar{D}_{T}\right)}\right)$. As the second step $\Delta t_{2}$ with respect to $t$ we take

$$
\Delta t_{2}:=\min \left\{\frac{1}{4 M\left(R_{1}\right) R_{1}}, \frac{1}{4 c\left(R_{1}\right) R_{1}}\right\},
$$

where

$$
R_{1}:=1+2 T M\left(R_{*}\right) R_{*}+\|F\|_{C\left(\bar{D}_{T}\right)}, \quad F:=\left(F_{1}, F_{2}, F_{3}\right) .
$$

Rewrite the system of equations (4.1) for $t \in\left[T_{*}, T_{*}+\Delta t_{2}\right]$ in the form of one vector equation

$$
V=\Psi V
$$

where operator $\Psi:=\left(\Psi_{1}, \Psi_{2}, \Psi_{3}\right)$ acts by formula

$$
\left\{\begin{array}{l}
\left(\Psi_{1} V\right)(x, t)=-\frac{1}{2} \int_{\alpha_{1}\left(x, t, \Delta t_{1}\right)}^{t} g\left(P_{\tau}, v_{3}\left(P_{\tau}\right)\right)\left(v_{1}\left(P_{\tau}\right)+v_{2}\left(P_{\tau}\right)\right) d \tau \\
\quad+\frac{1}{2} \int_{\alpha_{2}\left(x, t, \Delta t_{1}\right)}^{t} g\left(Q_{\tau_{0}}, v_{3}\left(Q_{\tau_{0}}\right)\right)\left(v_{1}\left(Q_{\tau_{0}}\right)+v_{2}\left(Q_{\tau_{0}}\right)\right) d \tau+F_{1, \Delta t_{1}}(x, t) \\
\left(\Psi_{2} V\right)(x, t)=-\frac{1}{2} \int_{\alpha_{3}\left(x, t, \Delta t_{1}\right)}^{t} g\left(Q_{\tau}, v_{3}\left(Q_{\tau}\right)\right)\left(v_{1}\left(Q_{\tau}\right)+v_{2}\left(Q_{\tau}\right)\right) d \tau \\
\quad+F_{2, \Delta t_{1}}(x, t), \\
\left(\Psi_{3} V\right)(x, t)=\int_{\alpha_{3}\left(x, t, \Delta t_{1}\right)}^{t} v_{1}\left(Q_{\tau}\right) d \tau+F_{3, \Delta t_{1}}(x, t)
\end{array}\right.
$$

First we show that operator $\Psi$ maps the ball $B\left(\left[T_{1}, T_{2}\right] ; R_{1}\right):=\{V \in$ $\left.C\left(\bar{D}_{T_{1}, T_{2}}\right):\|V\|_{C\left(\bar{D}_{T_{1}, T_{2}}\right)} \leq R_{1}\right\}$ into itself, where $T_{1}=T_{*}$ and $T_{2}=T_{*}+$ $\Delta t_{2}, \quad \bar{D}_{T_{1}, T_{2}}:=\bar{D} \cap\left\{T_{1} \leq t \leq T_{2}\right\}$.

Indeed, due to (3.14), (4.2)-(4.5) and (4.7) we have

$$
\begin{aligned}
\left\|\Psi_{1} V\right\|_{C\left(\bar{D}_{T_{1}, T_{2}}\right)} & \leq 2 M\left(R_{1}\right) R_{1} \Delta t_{2}+2 M\left(R_{*}\right) R_{*} \Delta t_{1}+\left\|F_{1}\right\|_{C\left(\bar{D}_{T}\right)} \\
& \leq 2^{-1}+2 T M\left(R_{*}\right) R_{*}+\|F\|_{C\left(\bar{D}_{T}\right)} \leq R_{1} .
\end{aligned}
$$

Analogously: $\left\|\Psi_{i} V\right\|_{C\left(\bar{D}_{T_{1}, T_{2}}\right)} \leq R_{1}, i=2,3$, and finally we receive that

$$
\|\Psi V\|_{C\left(\bar{D}_{T_{1}, T_{2}}\right)} \leq R_{1}
$$


Now let us show that operator $\Psi$ is a contracted mapping in this ball. Indeed, for $(x, t) \in \bar{D}_{T_{1}, T_{2}}$ due to (3.14), (4.4) and (4.7) we have

$$
\begin{aligned}
\mid\left(\Psi_{1}\right. & \left.V^{2}-\Psi_{1} V^{1}\right)(x, t) \mid \\
\leq & \frac{1}{2} \int_{\alpha_{1}\left(x, t, \Delta t_{1}\right)}^{t}\left(\left|g\left(P_{\tau}, v_{3}^{2}\left(P_{\tau}\right)\right)-g\left(P_{\tau}, v_{3}^{1}\left(P_{\tau}\right)\right)\right| \mid\left(v_{1}^{2}\left(P_{\tau}\right)+v_{2}^{2}\left(P_{\tau}\right) \mid\right.\right. \\
& \left.+\left|g\left(P_{\tau}, v_{3}^{1}\left(P_{\tau}\right)\right)\right|\left|v_{1}^{2}\left(P_{\tau}\right)-v_{1}^{1}\left(P_{\tau}\right)+v_{2}^{2}\left(P_{\tau}\right)-v_{2}^{1}\left(P_{\tau}\right)\right|\right) d \tau \\
& +\frac{1}{2} \int_{\alpha_{1}\left(x, t, \Delta t_{1}\right)}^{t}\left(\left|g\left(Q_{\tau_{0}}, v_{3}^{2}\left(Q_{\tau_{0}}\right)\right)-g\left(Q_{\tau_{0}}, v_{3}^{1}\left(Q_{\tau_{0}}\right)\right)\right| \mid\left(v_{1}^{2}\left(Q_{\tau_{0}}\right)+v_{2}^{2}\left(Q_{\tau_{0}}\right) \mid\right.\right. \\
& \left.+\left|g\left(Q_{\tau_{0}}, v_{3}^{1}\left(Q_{\tau_{0}}\right)\right) \| v_{1}^{2}\left(Q_{\tau_{0}}\right)-v_{1}^{1}\left(Q_{\tau_{0}}\right)+v_{2}^{2}\left(Q_{\tau_{0}}\right)-v_{2}^{1}\left(Q_{\tau_{0}}\right)\right|\right) d \tau \\
\leq & 2 c\left(R_{1}\right) R_{1} \Delta t_{2}\left\|v_{3}^{2}-v_{3}^{1}\right\|_{C\left(\bar{D}_{T_{1}, T_{2}}\right)}+2 M\left(R_{1}\right) \Delta t_{2}\left\|V^{2}-V^{1}\right\|_{C\left(\bar{D}_{T_{1}, T_{2}}\right)} \\
\leq & \frac{1}{2}\left\|v_{3}^{2}-v_{3}^{1}\right\|_{C\left(\bar{D}_{T_{1}, T_{2}}\right)}+\frac{1}{2 R_{1}}\left\|V^{2}-V^{1}\right\|_{C\left(\bar{D}_{T_{1}, T_{2}}\right)} \\
\leq & \left(\frac{1}{2}+\frac{1}{2 R_{1}}\right)\left\|V^{2}-V^{1}\right\|_{C\left(\bar{D}_{T_{1}, T_{2}}\right)}=q_{1}\left\|V^{2}-V^{1}\right\|_{C\left(\bar{D}_{T_{1}, T_{2}}\right)},
\end{aligned}
$$

where $q_{1}:=\frac{1}{2}\left(1+\frac{1}{R_{1}}\right)<1$, since $R_{1}>1$ in view of (4.5).

Analogously we receive, that

$$
\begin{aligned}
& \left|\left(\Psi_{i} V^{2}-\Psi_{i} V^{1}\right)(x, t)\right| \leq q_{i}\left\|V^{2}-V^{1}\right\|_{C\left(\bar{D}_{T_{1}, T_{2}}\right)}, \\
& 0<q_{i}:=\text { const }<1, \quad i=2,3 .
\end{aligned}
$$

Thus, due to the theorem about contracted mapping it follows the solvability of vector equation (4.6) in space $C\left(\bar{D}_{T_{1}, T_{2}}\right)$.

Continuing this process step by step, and taking into account the fact that in view of global a priori estimate (4.3), and also (2.28), the length of each step $\Delta t_{i}$ does not depend on $i$. Thus we receive the global solvability of the system of equations (2.22), and therefore of problem (1.1), (1.2) in domain $D_{T}$ for any $T>0$.

Remark 4.1. One may present the examples of functions $g=g(x, t, s)$, satisfying the conditions of the Theorem 4.1, or, the same, the conditions (2.1), (3.13) and (3.14). Such function is

$$
g(x, t, s)=\sum_{k=1}^{n} \alpha_{k}(x, t)|s|^{\beta_{k}},
$$

where $\alpha_{k} \in C\left(\bar{D}_{\infty}\right), k=1, \ldots, n ; \alpha_{1}(x, t)>0$ for $(x, t) \in \bar{D}_{\infty}$ and $\beta_{1}>$ $\beta_{i} \geq 1, i=2, \ldots, n$; and also function $g(x, t, s)=\alpha(x, t) g_{0}(s)$, where $\alpha \in$ $C\left(\bar{D}_{\infty}\right), g_{0} \in \operatorname{Lip}_{l o c}(\mathbb{R})$ and $\liminf \operatorname{sis}_{|s| \rightarrow+\infty} g_{0}(s)>-\infty$, satisfy the conditions of Theorem 4.1. 


\section{Uniqueness of the solution of the problem (1.1), (1.2)}

Lemma 5.1. Let the conditions (3.13), (3.14) be fulfilled. Then for any $T>0$ the problem (1.1), (1.2) cannot have more than one strong generalized solution of the class $C^{1}$ in domain $D_{T}$.

Proof. Indeed, suppose that the problem (1.1), (1.2) has two different possible strong generalized solutions $u^{1}$ and $u^{2}$ of the class $C^{1}$ in the domain $D_{T}$. Then, according to Definition 1.1 there exists the sequence of functions $u_{n}^{i} \in \stackrel{\circ}{C}^{2}\left(\bar{D}_{T}, \Gamma_{T}\right)$, such that

$$
\begin{gathered}
\lim _{n \rightarrow \infty}\left\|u_{n}^{i}-u^{i}\right\|_{C^{1}\left(\bar{D}_{T}\right)}=0, \quad \lim _{n \rightarrow \infty}\left\|L u_{n}^{i}-f\right\|_{C\left(\bar{D}_{T}\right)}=0, \\
\lim _{n \rightarrow \infty}\left\|g\left(x, t, u_{n}^{i}\right) u_{n t}^{i}-g\left(x, t, u^{i}\right) u_{t}^{i}\right\|_{C\left(\bar{D}_{T}\right)}=0, \quad i=1,2 .
\end{gathered}
$$

Let us use well-known notation $\square:=\partial^{2} / \partial t^{2}-\partial^{2} / \partial x^{2}$ and assume that $\omega_{n}:=u_{n}^{2}-u_{n}^{1}$. It is easy to see that function $\omega_{n} \in \stackrel{\circ}{C}^{2}\left(\bar{D}_{T}, \Gamma_{T}\right)$ is a classical solution of the following problem

$$
\begin{gathered}
\square \omega_{n}+g_{n}=f_{n}, \\
\left.\omega_{n}\right|_{\Gamma_{T}}=0,
\end{gathered}
$$

where

$$
g_{n}:=g\left(x, t, u_{n}^{2}\right) u_{n t}^{2}-g\left(x, t, u_{n}^{1}\right) u_{n t}^{1}, \quad f_{n}:=L u_{n}^{2}-L u_{n}^{1} .
$$

Due to the first equality from (5.1) there exists the number $A:=$ const $>$ 0 , not dependent on indices $i$ and $n$, such that

$$
\left\|u_{n}^{i}\right\|_{C^{1}\left(\bar{D}_{T}\right)} \leq A
$$

According to the second equalities from (5.1) and (5.4) it follows that

$$
\lim _{n \rightarrow \infty}\left\|f_{n}\right\|_{C\left(\bar{D}_{T}\right)}=0 \text {. }
$$

From (3.14), (5.5) and the first equality of (5.4) it is clear that

$$
\begin{aligned}
g_{n}^{2} & =\left(g\left(x, t, u_{n}^{2}\right) \omega_{n t}+\left[g\left(x, t, u_{n}^{2}\right)-g\left(x, t, u_{n}^{1}\right)\right] u_{n t}^{1}\right)^{2} \\
& \leq 2 M^{2}(A) \omega_{n t}^{2}+2 A^{2} c^{2}(A) \omega_{n}^{2} .
\end{aligned}
$$

Multiplying the both sides of (5.2) by $\omega_{n t}$ and integrating the received equality in the domain $D_{\tau}$, due to boundary conditions (5.3), as it was in receiving of (2.11) from (2.5), (2.6) we shall have

$$
w_{n}(\tau):=\int_{\Omega_{\tau}}\left(\omega_{n x}^{2}+\omega_{n t}^{2}\right) d x=2 \int_{D_{\tau}}\left(f_{n}-g_{n}\right) \omega_{n t} d x d t .
$$


Due to estimate (5.7) and the inequality of Cauchy we shall have

$$
\begin{aligned}
& 2 \int_{D_{\tau}}\left(f_{n}-g_{n}\right) \omega_{n t} d x d t \leq \int_{D_{\tau}}\left(f_{n}-g_{n}\right)^{2} d x d t+\int_{D_{\tau}} \omega_{n t}^{2} d x d t \\
& \quad \leq 2 \int_{D_{\tau}} f_{n}^{2} d x d t+2 \int_{D_{\tau}} g_{n}^{2} d x d t+\int_{D_{\tau}} \omega_{n t}^{2} d x d t \\
& \quad \leq 2 \int_{D_{\tau}} f_{n}^{2} d x d t+4 A^{2} c^{2}(A) \int_{D_{\tau}} \omega_{n}^{2} d x d t+\left[1+4 M^{2}(A)\right] \int_{D_{\tau}} \omega_{n t}^{2} d x d t .
\end{aligned}
$$

Further, from equality $\omega_{n}(x, t)=\int_{x}^{t} \omega_{n t}(x, \tau) d \tau,(x, t) \in \bar{D}_{T}$, which follows from (5.3), by use of standard considerations we receive inequality $[25$, p. 63$]$

$$
\int_{D_{\tau}} \omega_{n}^{2} d x d t \leq \tau^{2} \int_{D_{\tau}} \omega_{n t}^{2} d x d t
$$

From (5.8)-(5.10) it follows that

$$
\begin{aligned}
w_{n}(\tau) & \leq\left[1+4 M^{2}(A)+4 \tau^{2} A^{2} c^{2}(A)\right] \int_{D_{\tau}} \omega_{n t}^{2} d x d t+2 \int_{D_{\tau}} f_{n}^{2} d x d t \\
& \leq\left[1+4 M^{2}(A)+4 T^{2} A^{2} c^{2}(A)\right] \int_{0}^{\tau} w_{n}(\sigma) d \sigma+2 \int_{D_{T}} f_{n}^{2} d x d t .
\end{aligned}
$$

Whence by the Gronwall's lemma we receive that

$$
w_{n}(\tau) \leq c_{2}\left\|f_{n}\right\|_{L_{2}\left(D_{T}\right)}^{2}, \quad 0<\tau \leq T
$$

where $c_{2}:=2 \exp \left[1+4 M^{2}(A)+4 T^{2} A^{2} c^{2}(A)\right] T$.

Conducting the same considerations, as those used for receiving of (2.13), and taking into account obvious inequality

$$
\left\|f_{n}\right\|_{L_{2}\left(D_{T}\right)}^{2} \leq\left\|f_{n}\right\|_{C\left(\bar{D}_{T}\right)}^{2} \text { mes } D_{T}
$$

and also due to (5.11), for $(x, t) \in \bar{D}_{T}$ we have

$$
\left|\omega_{n}(x, t)\right|^{2} \leq t w_{n}(t) \leq T c_{2} \text { mes } D_{T}\left\|f_{n}\right\|_{C\left(\bar{D}_{T}\right)}^{2}=\frac{c_{2} T^{3}}{2}\left\|f_{n}\right\|_{C\left(\bar{D}_{T}\right)}^{2} .
$$

From this inequality it follows immediately that

$$
\left\|\omega_{n}\right\|_{C\left(\bar{D}_{T}\right)} \leq T \sqrt{\frac{c_{2} T}{2}}\left\|f_{n}\right\|_{C\left(\bar{D}_{T}\right)} .
$$

Recalling the definition of function $\omega_{n}$, according to the first equality from (5.1) we have $\lim _{n \rightarrow \infty}\left\|\omega_{n}\right\|_{C^{1}\left(\bar{D}_{T}\right)}=\left\|u^{2}-u^{1}\right\|_{C^{1}\left(\bar{D}_{T}\right)}$ and all the more

$$
\lim _{n \rightarrow \infty}\left\|\omega_{n}\right\|_{C\left(\bar{D}_{T}\right)}=\left\|u^{2}-u^{1}\right\|_{C\left(\bar{D}_{T}\right)}
$$

Due to this equality and (5.6), passing in (5.12) to limit for $n \rightarrow \infty$ we receive $\left\|u^{2}-u^{1}\right\|_{C\left(\bar{D}_{T}\right)}=0$, i.e. $u^{1}=u^{2}$, which proves Lemma 5.1. 


\section{The case of nonexistence of a global solution of the problem (1.1), (1.2)}

Below we will show that violation of condition (2.1) may cause the absence of global solvability of the problem (1.1), (1.2) in the sense of Definition 1.2. Indeed, let $g(x, t, s)=-|s|^{\alpha} s, s \in \mathbb{R}, \alpha>-1$.

Lemma 6.1. Let $u$ be a strong generalized solution of the problem (1.1), (1.2) of the class $C^{1}$ in the domain $D_{T}$ in the sense of Definition 1.1. Then it is valid the following integral equality

$$
\int_{D_{T}} u \square \varphi d x d t=\int_{D_{T}}|u|^{\alpha} u u_{t} \varphi d x d t+\int_{D_{T}} f \varphi d x d t
$$

for any function $\varphi$, such that

$$
\varphi \in C^{2}\left(\bar{D}_{T}\right),\left.\quad \varphi\right|_{t=T}=0,\left.\quad \varphi_{t}\right|_{t=T}=0,\left.\quad \varphi\right|_{\gamma_{2, T}}=0 .
$$

Proof. According to the definition of a strong generalized solution $u$ of the problem (1.1), (1.2) of the class $C^{1}$ in the domain $D_{T}$, function $u \in C^{1}\left(\bar{D}_{T}\right)$ and there exists the sequence of functions $u_{n} \in \stackrel{\circ}{C}^{2}\left(\bar{D}_{T}, \Gamma_{T}\right)$, such that the equalities of (2.3) and (2.4) for $g(x, t, s)=-|s|^{\alpha} s, s \in \mathbb{R}, \alpha>-1$ are valid.

Suppose that $f_{n}:=L u_{n}$. Let us multiply the both sides of equality $L u_{n}=$ $f_{n}$ by function $\varphi$ and integrate the received equality in the domain $D_{T}$. As a result of integration by parts of the left-hand side of this equality, due to (6.2) and boundary conditions $(2.6)$ we receive

$$
\int_{D_{T}} u_{n} \square \varphi d x d t=\int_{D_{T}}\left|u_{n}\right|^{\alpha} u_{n} u_{n t} \varphi d x d t+\int_{D_{T}} f_{n} \varphi d x d t .
$$

By passing to limit in this equality for $n \rightarrow \infty$, according to (2.3)-(2.5) we receive (6.1). Thus Lemma 6.1 is proved.

Let us introduce into consideration function $\varphi^{0}:=\varphi^{0}(x, t)$ such that $[9$, pp. 10-12]

$$
\varphi^{0} \in C^{2}\left(\bar{D}_{\infty}\right), \quad \varphi^{0}+\varphi_{t}^{0} \leq 0,\left.\quad \varphi^{0}\right|_{D_{T=1}}>0,\left.\quad \varphi^{0}\right|_{\gamma_{2, \infty}}=0,\left.\quad \varphi^{0}\right|_{t \geq 1}=0
$$

and

$$
\kappa_{0}:=\int_{D_{T=1}} \frac{\left|\square \varphi^{0}\right|^{p^{\prime}}}{\left|\varphi^{0}\right|^{p^{\prime}-1}} d x d t<+\infty, \quad p^{\prime}=\frac{\alpha+2}{\alpha+1} .
$$

It is easy to verify that in the role of function $\varphi^{0}$, satisfying conditions (6.3) and (6.4), one may use function

$$
\varphi^{0}(x, t)= \begin{cases}x^{n}(1-t)^{m}, & (x, t) \in D_{T=1}, \\ 0, & t \geq 1,\end{cases}
$$

for sufficiently large positive numbers $n$ and $m$. 
Suppose that $\varphi_{T}(x, t):=\varphi^{0}\left(\frac{x}{T}, \frac{t}{T}\right), T>0$. Due to $(6.3)$ it is easy to see that

$$
\begin{gathered}
\varphi_{T} \in C^{2}\left(\bar{D}_{T}\right), \quad \varphi_{T}+T \varphi_{T t} \leq 0,\left.\quad \varphi_{T}\right|_{D_{T}}>0,\left.\quad \varphi_{T}\right|_{\gamma_{2, T}}=0, \\
\left.\varphi_{T}\right|_{t=T}=0,\left.\quad \varphi_{T t}\right|_{t=T}=0 .
\end{gathered}
$$

Supposing that function $f$ is fixed, let us introduce into consideration a function of one variable $T$

$$
\zeta(T):=\int_{D_{T}} f \varphi_{T} d x d t, \quad T>0 .
$$

The following theorem on the nonexistence of a global solution of the problem (1.1), (1.2) is valid.

Theorem 6.1. Let $g(x, t, s)=-|s|^{\alpha} s, s \in \mathbb{R}, \alpha>-1, f \in C\left(\bar{D}_{\infty}\right)$ and $f \geq 0$ in the domain $D_{\infty}$. If

$$
\liminf _{T \rightarrow+\infty} \zeta(T)>0
$$

then there exists positive number $T^{*}:=T^{*}(f)$, such that for $T>T^{*}$ the problem (1.1), (1.2) cannot have a strong generalized solution $u$ of the class $C^{1}$ in the domain $D_{T}$.

Proof. Suppose, that in conditions of this theorem there exists a strong generalized solution $u$ of the problem (1.1), (1.2) of the class $C^{1}$ in the domain $D_{T}$. Then according to Lemma 6.1 equality (6.1) holds, where due to (6.5) in the role of function $\varphi$ can be taken function $\varphi=\varphi_{T}$, i.e.

$$
\int_{D_{T}} u \square \varphi_{T} d x d t=\int_{D_{T}}|u|^{\alpha} u u_{t} \varphi_{T} d x d t+\int_{D_{T}} f \varphi_{T} d x d t
$$

In view of (1.2) and (6.5) we have

$$
\begin{aligned}
\int_{D_{T}}|u|^{\alpha} u u_{t} \varphi_{T} d x d t & =\frac{1}{\alpha+2} \int_{D_{T}} \varphi_{T}\left(|u|^{\alpha+2}\right)_{t} d x d t=-\frac{1}{\alpha+2} \int_{D_{T}}|u|^{\alpha+2} \varphi_{T t} d x d t \\
& \geq \frac{1}{(\alpha+2) T} \int_{D_{T}}|u|^{\alpha+2} \varphi_{T} d x d t .
\end{aligned}
$$

Due to this inequality and (6.6) from (6.8) it follows, that

$$
\frac{1}{p T} \int_{D_{T}}|u|^{p} \varphi_{T} d x d t \leq \int_{D_{T}} u \square \varphi_{T} d x d t-\zeta(T), \quad p:=\alpha+2>1 .
$$

If in Young equality with parameter $\varepsilon>0, a b \leq \frac{\varepsilon}{p} a^{p}+\frac{1}{p^{\prime} \varepsilon^{p^{\prime}-1}} b^{p^{\prime}} ; a, b \geq$ $0, \frac{1}{p}+\frac{1}{p^{\prime}}=1, p>1$ we shall take $a=|u| \varphi_{T}^{\frac{1}{p}}, b=\frac{\left|\square \varphi_{T}\right|}{\varphi_{T}^{\frac{1}{p}}}, \varepsilon=\frac{1}{T}$, then since $\frac{p^{\prime}}{p}=p^{\prime}-1$ we obtain

$$
\left|u \square \varphi_{T}\right|=|u| \varphi_{T}^{\frac{1}{p}} \frac{\left|\square \varphi_{T}\right|}{\varphi_{T}^{\frac{1}{p}}} \leq \frac{1}{p T}|u|^{p} \varphi_{T}+\frac{T^{p^{\prime}-1}}{p^{\prime}} \frac{\left|\square \varphi_{T}\right|^{p^{\prime}}}{\varphi_{T}^{p^{\prime}-1}} .
$$


According to this inequality and (6.9) we have

$$
0 \leq \frac{T^{p^{\prime}-1}}{p^{\prime}} \int_{D_{T}} \frac{\left|\square \varphi_{T}\right|^{p^{\prime}}}{\varphi_{T}^{p^{\prime}-1}} d x d t-\zeta(T)
$$

Due to $(6.3),(6.4)$, after changing variables $x=T x_{1}, t=T t_{1}$, it is easy to verify that

$$
\int_{D_{T}} \frac{\left|\square \varphi_{T}\right|^{p^{\prime}}}{\varphi_{T}^{p^{\prime}-1}} d x d t=\frac{1}{T^{2\left(p^{\prime}-1\right)}} \int_{D_{T=1}} \frac{\left|\square \varphi^{0}\right|^{p^{\prime}}}{\left|\varphi^{0}\right|^{p^{\prime}-1}} d x_{1} d t_{1}=\frac{\kappa_{0}}{T^{2\left(p^{\prime}-1\right)}} .
$$

In view to this from $(6.10)$ we receive

$$
0 \leq \frac{\kappa_{0}}{p^{\prime} T^{p^{\prime}-1}}-\zeta(T)
$$

Since $p^{\prime}=\frac{p}{p-1}>1$ due to (6.4) we have

$$
\lim _{T \rightarrow+\infty} \frac{\kappa_{0}}{p^{\prime} T^{p^{\prime}-1}}=0 .
$$

Therefore, by virtue of (6.7) there exists positive number $T^{*}:=T^{*}(f)$, such that for $T>T^{*}$ the right-hand side of (6.11) will be negative, whereas the left-hand side of this inequality is zero. This means that if there exists a strong generalized solution $u$ of the problem (1.1), (1.2) of the class $C^{1}$ in the domain $D_{T}$, then necessarily $T \leq T^{*}$, which proves the Theorem 6.1 .

Remark 6.1. It is easy to verify that if $f \in C\left(\bar{D}_{\infty}\right), f \geq 0$ and $f(x, t) \geq c t^{-m}$ for $t \geq 1$, where $c:=$ const $>0,0 \leq m:=$ const $\leq 2$, then condition (6.7) will be fulfilled, and so for $g(x, t, s)=-|s|^{\alpha} s, s \in \mathbb{R}, \alpha>-1$ the problem (1.1), (1.2) for sufficiently large $T$ will not have a strong generalized solution $u$ of the class $C^{1}$ in the domain $D_{T}$.

Indeed, let us introduce in (6.6) the transformation of independent variables $x$ and $t$ by formula $x=T x_{1}, t=T t_{1}$, after some estimations we have

$$
\begin{aligned}
& \zeta(T)=T^{2} \int_{D_{T=1}} f\left(T x_{1}, T t_{1}\right) \varphi^{0}\left(x_{1}, t_{1}\right) d x_{1} d t_{1} \\
& \geq c T^{2-m} \int_{D_{T=1} \cap\left\{t_{1} \geq T^{-1}\right\}} t_{1}^{-m} \varphi^{0}\left(x_{1}, t_{1}\right) d x_{1} d t_{1} \\
& \quad+T^{2} \int_{D_{T=1} \cap\left\{t_{1}<T^{-1}\right\}} f\left(T x_{1}, T t_{1}\right) \varphi^{0}\left(x_{1}, t_{1}\right) d x_{1} d t_{1}
\end{aligned}
$$

in supposition that $T>1$. Further, let $T_{1}>1$ be any fixed number. Then from the last inequality for function $\zeta$ we have

$$
\begin{aligned}
\zeta(T) & \geq c T^{2-m} \int_{D_{T=1} \cap\left\{t_{1} \geq T^{-1}\right\}} t_{1}^{-m} \varphi^{0}\left(x_{1}, t_{1}\right) d x_{1} d t_{1} \\
& \geq c \int_{D_{T=1} \cap\left\{t_{1} \geq T_{1}^{-1}\right\}} t_{1}^{-m} \varphi^{0}\left(x_{1}, t_{1}\right) d x_{1} d t_{1},
\end{aligned}
$$

if $T \geq T_{1}>1$. From the latter inequality immediately follows the validity of $(6 . \overline{7})$. 


\section{References}

[1] Lions, J.L., Strauss, W.: Some nonlinear evolution equations. Bull. Soc. Math. France 93, 43-96 (1965)

[2] Lions, J.L.: Quelques méthodes de résolution des problémes aux limites non linéaires. Dunod, Gauthier-Villars, Paris (1969)

[3] Bitsadze, A.V.: Some Classes of Partial Differential Equations, Nauka, Moscow (1981, in Russian)

[4] Strauss, W.: Nonlinear Invariant Wave Equations. Lecture Notes in Physics, vol. 78. Springer, Berlin (1978)

[5] Ebihara, Y.: On the global Cauchy problem for $\square u+g^{2} u-u^{3}=f$. J. Math. Anal. Appl. 64, 398-406 (1978)

[6] Nakao, M., Ono, K.: Existence of global solutions to the Cauchy problem for the semilinear dissipative wave equations. Math. Z. 214, 325-342 (1993)

[7] Hörmander, L.: Lectures on Nonlinear Hyperbolic Differential Equations. Springer, Berlin (1997)

[8] Ono, K.: On the global existence and decay of solutions for semilinear telegraph equations. Int. J. Appl. Math. 2(9), 1121-1136 (2000)

[9] Mitidieri, E., Pohozaev, S.I.: A Priori Estimates and Blow-Up of Solutions to Nonlinear Partial Differential Equations and Inequalities. Proc. V. A. Steklov Inst. Math. vol. 234 (2001)

[10] Veron, L., Pohozaev, S.I.: Blow-up results for nonlinear hyperbolic inequalities. Ann. Scuola Norm. Super. Pisa. Cl. Sci. Ser. 4. 29(2), 393-420 (2001)

[11] Witelski, T.P., Bernoff, A.J., Bertozzi, A.L.: Blow-up and dissipation in a critical case unstable thin film equation. Eur. J. Appl. Math. 15(2), 223-256 (2004)

[12] Todorova, G., Vitillaro, E.: Blow-up for nonlinear dissipative wave equations in $R^{n}$. J. Math. Anal. Appl. 303(1), 242-257 (2005)

[13] Liu, L., Wang, M.: Global existence and blow-up of solutions for some hyperbolic systems with damping and source terms. J. Nonlinear Anal. Theory Methods Appl. 64, 69-91 (2006)

[14] Kharibegashvili, S.: On the solvability of one multidimensional version of the first Darboux problem for some nonlinear wave equations. J. Nonlinear Anal. Theory Methods Appl. 68, 912-924 (2008)

[15] Kharibegashvili, S.: On the solvability of the Cauchy characteristic problem for a nonlinear equation with iterated wave operator in the principal part. J. Math. Anal. Appl. 338, 71-84 (2008)

[16] Jokhadze, O., Midodashvili, B.: The first Darboux problem for nonlinear wave equations with a nonlinear positive source term. J. Nonlinear Anal. Theory Methods Appl. 69, 3005-3015 (2008) 
[17] Jokhadze, O.: On existence and nonexistence of global solutions of Cauchy-Goursat problem for nonlinear wave equations. J. Math. Anal. Appl. 340, 1033-1045 (2008)

[18] Jokhadze, O.: The Cauchy-Goursat problem for one-dimensional semilinear wave equations. Commun. Partial Differ. Equ. 34(4), 367-382 (2009)

[19] Goursat, E.: The Course of Mathematical Analysis, vol. 3, Part I (1933)

[20] Henry, D.: Geometrical Theory of Semi-Linear Parabolic Equations. Mir, Moscow (1985)

[21] Hörmander, L.: Linear Partial Differential Operators. Springer, Berlin (1963)

[22] Whitney, H.: Analytic extension of differentiable functions defined in closed set. Trans. Am. Math. Soc. 36, 63-89 (1934)

[23] Hestenes, M.R.: Extensions of de range of a differentiable function. Duke Math. J. 8, 183-192 (1941)

[24] Trenogin, V.A.: Functional Analysis. Nauka, Moscow (1993, in Russian)

[25] Ladyzhenskaya, O.A.: Boundary Value Problems of Mathematical Physics. Nauka, Moscow (1973, in Russian)

Otar Jokhadze

I. Javakhishvili Tbilisi State University

2, University Str.

0186 Tbilisi

Georgia

e-mail: ojokhadze@yahoo.com

Received: 13 May 2011.

Accepted: 3 May 2012. 